\title{
Buscando a Nemo...: la organización y recuperación de la información en los catálogos en línea destinados al público infantil
}

\author{
Yolanda Martín González \\ Ana B. Ríos Hilario \\ Universidad de Salamanca (España)
}

\section{Resumen}

La recuperación de la información constituye un elemento crucial para el usuario de las unidades de información. Pese a que siempre se ha dedicado un mayor estudio a los procesos de búsqueda del público adulto, el infantil constituye un importante foco de atención para los profesionales que trabajan en las secciones especializadas en este grupo de usuarios, aunque muy pocas veces se han plasmado por escrito sus impresiones. Con nuestro trabajo pretendemos crear un esquema que permita la evaluación posterior de los catálogos en línea dirigidos al público infantil, tomando como base los estudios previos existentes en la materia, sobre todo los destinados al público adulto. Para llevar a cabo nuestro trabajo, después de una breve introducción a los catálogos en línea, pasamos a analizar los estudios más importantes existentes sobre esta materia, que establecen métodos de evaluación fundamentalmente para los OPAC en general. Posteriormente, desarrollamos la creación del modelo propiamente dicho, centrándonos en dos aspectos fundamentales: las prestaciones de búsqueda y las características de la interfaz. Ateniéndonos a estos parámetros podemos decir que el catálogo en línea destinado a los usuarios infantiles debería cumplir al menos las siguientes condiciones: ser atractivo, comprensible y manejable.

Palabras clave: Recuperación de información. OPAC. Bibliotecas infantiles. Niños.

\begin{abstract}
Information retrieval constitutes a crucial element for the user of information units. In spite of the fact that more attention has been paid to mature public's search, children constitute an important focus of attention for the professionals that work in the sections specialized in this group of users, although their impressions have hardly ever been registered in writing. We seek to create an outline that allows the later evaluation of on-line catalogs addressed to children, taking as a background the previous studies on the matter, mainly those dedicated to the mature
\end{abstract}

Scire. $12: 1$ (en.-jun. 2006) 153-162. ISSN 1135-3761. 
public. After a brief introduction to on-line catalogs, the more important studies on the evaluation of OPACs in general are analyzed. Later, two fundamental aspects are considered in detail: search features and interface characteristics. As a conclusion, on-line catalogs aimed at children should fulfill at least the following conditions: attractiveness, comprehensibility and usability.

Keywords: Information retrieval. OPAC. Children libraries. Children.

\section{Introducción}

El catálogo constituye hoy por hoy un instrumento básico para la recuperación de información al tiempo que se erige en el eje informativo en el ámbito bibliotecario, esto es, en el "corazón" del sistema de localización y recuperación de información.

En el momento actual, el grupo de usuarios al que se dirige se ha visto aumentado y diversificado. Igualmente, las necesidades que presenta el público de los catálogos automatizados son actualmente más variadas que nunca, al igual que sus habilidades generales y capacidades especializadas para la recuperación de información (edad, cultura, adaptación tecnológica, etcétera). De este modo, el diseñador del sistema se ve obligado a tener en cuenta los distintos tipos y niveles de usuarios.

Los responsables del diseño de OPAC han solventado parte de los problemas mencionados incorporando en este tipo de catálogos herramientas tales como los mensajes generales explicativos, los que indican lo que se ha hecho en los pasos anteriores durante una búsqueda, los que indican qué opciones están disponibles para la acción siguiente del usuario y cómo debe ejecutarlas, los visualizados en respuestas a una orden no válida introducida por el usuario, pantallas de ayuda y ayudas en línea.

La mayor parte de los estudios realizados sobre visualización y presentación de la información en OPAC se basan en el estudio y análisis de sus interfaces de usuario. En particular, en la literatura sobre evaluación de catálogos automatizados - y más en concreto de su amigabilidad — suele ser común la aplicación de dos parámetros: las prestaciones de búsqueda a las que da acceso la interfaz y las características propias de dicha interfaz: diseño, ergonomía y amigabilidad.

Es importante resaltar algo que hoy ya nadie pone en duda, y es que el entorno Web, soporte para muchos catálogos en línea, ha supuesto, por un lado, una importante revolución en cuanto al acceso y a la recuperación de la información al combinar ambas tareas; por otro, ha mejorado significativamente la amigabilidad de los OPAC al adoptar sistemas de navegación y presentación familiares a los usuarios, evitando el uso de otros lenguajes de consulta más complejos (por ejemplo, comandos) y aprovechando las oportunidades de visualización del hipertexto. 
El objetivo de nuestro estudio consiste en crear un modelo de evaluación de catálogos automatizados destinados a un grupo de usuarios concreto: los niños. Dado que bajo este término puede hacerse referencia a un conjunto excesivamente amplio de individuos con capacidades, habilidades y necesidades diferentes, nos centraremos en los niños con edades comprendidas entre los 6 y los 14 años, edad en la que generalmente las bibliotecas establecen la barrera entre el público infantil y el adulto.

Debido a la carencia de bibliografía especializada sobre el tema, el modelo que proponemos toma como base los estudios realizados hasta el momento en nuestro país sobre la evaluación de los catálogos en línea de acceso público en general. Sobresalen entre dichos estudios los realizados por las profesoras Blanca Rodríguez y $\mathrm{M}^{\mathrm{a}}$ Luisa Alvite y los llevados a cabo por Purificación Moscoso. En todos ellos, las autoras examinan dos aspectos complementarios: en primer lugar, las prestaciones que ofrecen estos sistemas en cuanto a la recuperación y localización de la información deseada; en segundo lugar, su aceptación por parte de los diferentes usuarios.

\section{Principales requisitos para el diseño de un OPAC infantil}

Resulta a todas luces evidente que las necesidades informativas que presentan los niños no son las mismas que las de los adultos, como tampoco lo son sus formas de localizar el documento que les interesa. Por esta razón, a la hora de determinar cuáles son los aspectos que deberían estar contemplados en un catálogo en línea para el público infantil, debemos partir de las siguientes premisas:

- En primer lugar, debe decidirse si se establece o no un prototipo único de usuario ya que, según la definición que del término infancia ofrece el Diccionario de la Real Academia Española, este comprende el "periodo de la vida humana desde que se nace hasta la pubertad". Lógicamente, las capacidades y habilidades que para la búsqueda de información puede poseer un niño de 6 años no son las mismas que las que tiene un preadolescente.

- En segundo lugar, la mayor parte de los niños que utilizan el OPAC lo hace de un modo esporádico; por tanto, hay que evitar, en la medida de lo posible, la utilización de lenguajes de recuperación y optar por la utilización de menús o comandos que faciliten su consulta.

- En tercer lugar, es muy probable que el acceso al OPAC se haga a través de Internet, una herramienta con la que los niños se encuentran cada vez más familiarizados. Por esta razón, es aconsejable que el catálogo se asemeje tanto en su diseño como en su lenguaje al del entorno web.

Teniendo en cuenta las condiciones que acabamos de mencionar, y tomando como base los estudios ya citados, detallamos a continuación aquellos elementos 
que creemos deberían contemplar todos los catálogos automatizados destinados al público infantil, haciendo una distinción entre lo que se refiere a las prestaciones de búsqueda y las características que ha de poseer la interfaz de usuario.

\subsection{Prestaciones de búsqueda}

\subsubsection{Página de entrada}

\begin{tabular}{|l|c|}
\hline Información sobre el sistema y la base de datos & No \\
\hline Idiomas de acceso & Sí \\
\hline Niveles de búsqueda & Sí \\
\hline Acceso a distintos catálogos & Sí \\
\hline Buzón de sugerencias & Sí \\
\hline Registro de usuario & Sí \\
\hline Integración con la biblioteca & Sí \\
\hline Información sobre la ejecución de la búsqueda & Sí \\
\hline Acceso por lenguaje libre y/o controlado & Sí \\
\hline Estrategia de búsqueda y recuperación de la información & No \\
\hline Campos recuperables & No \\
\hline Uso de operadores & No \\
\hline Acceso y visualización de registros de autoridad & Sí \\
\hline Navegación por índices & Sí \\
\hline Nivel de hipertextualidad & Sí \\
\hline Información sobre el número de ítems & Sí \\
\hline Posibilidad de limitar la estrategia de búsqueda & No \\
\hline Posibilidad de iniciar una nueva búsqueda & Sí \\
\hline Historial de búsqueda & \\
\hline Búsqueda inteligente & \\
\hline
\end{tabular}

Tabla I. Prestaciones de búsqueda en página de entrada

Esta página ofrece al usuario una visión general de la información y los distintos recursos que puede encontrar en el catálogo. Sin embargo, cuando se trata de niños no es necesario que los datos aquí presentados sean exhaustivos.

Es importante que esta página de presentación (tabla I) proporcione, en primer lugar, la información en distintos idiomas, dada la multiculturalidad existente en nuestro país; en segundo lugar, se ha de permitir el acceso vía web de usuarios procedentes del exterior de la biblioteca. Es, igualmente, necesario ofrecer información sobre la manera en la que se puede ejecutar la estrategia de búsqueda y el establecimiento de distintos niveles adecuados a las diferentes necesidades de los 
usuarios en función de su edad al igual que el acceso a distintos catálogos. Esto puede ser útil sobre todo para los preadolescentes que utilizan la biblioteca para sus tareas escolares. Por otra parte, es imprescindible que se permita acometer la búsqueda a través del lenguaje libre. A los niños les resulta más fácil realizar la consulta utilizando su propio lenguaje, aunque creemos que el sistema sí ha de facilitar la localización de información ofreciendo, por ejemplo, una aclaración sobre el término que se está utilizando y sus distintas acepciones. Además, se ha de ofrecer la posibilidad de buscar por otros términos relacionados.

Con el fin de mejorar las prestaciones de este tipo de OPAC, es recomendable un buzón de sugerencias, ya que a través de él el diseñador del sistema y el profesional de la información pueden conocer las deficiencias que presenta el OPAC para los usuarios de la biblioteca.

El registro de usuario constituye un instrumento muy útil porque es un modo de que el niño se convierta en un ente autónomo en la biblioteca y pueda realizar determinadas tareas por sí mismo.

En lo relativo a las estrategias de búsqueda y recuperación de la información, en nuestra opinión, se han de ofrecer como campos recuperables título, autor, materia y cualquier campo que permita limitar la estrategia de búsqueda. La utilización de operadores puede complicar la localización de información más que facilitarla. Tampoco es necesario el acceso y visualización de registros de autoridad ni que se permita la navegación por índices.

La opción de iniciar una nueva búsqueda en cualquier punto debería estar presente en todos los catálogos, independientemente del tipo de usuario que lo utilice, puesto que entre otras cosas supone un importante ahorro de tiempo en la localización de la información. Sin embargo, creemos que no es necesario ofrecer el historial de búsquedas, ya que las que realizan los niños no suelen ser demasiado complejas.

Por lo que se refiere a la búsqueda inteligente, es importante que el sistema sea capaz de obviar determinados fallos gramaticales, muy frecuentes, por otra parte, en estos usuarios, es decir, que obvie la utilización indistinta de mayúsculas y minúsculas, posibilite el trunque cuando sea necesario y controle la sinonimia y la utilización de redes semánticas.

\subsubsection{Páginas intermedias}

\begin{tabular}{|l|c|}
\hline Información de ítems recuperados & Sí \\
\hline Posibilidad de ordenación de los resultados & No \\
\hline Posibilidad de mantener varias ventanas abiertas & No \\
\hline
\end{tabular}

Tabla II. Prestaciones de búsqueda en páginas intermedias

Scire. $12: 1$ (en.-jun. 2006) 153-162. ISSN 1135-3761. 
En estas páginas (tabla II) la información sobre el número de ítems asociados a una entrada puede resultar muy importante puesto que, en el caso de que sea numeroso, va a permitir al niño acotar su búsqueda. En cambio, consideramos prescindibles aspectos como son la posibilidad de ordenación de los resultados, de identificación de las páginas o de mantener varias ventanas abiertas - en concreto, esta última opción puede dificultar más que ayudar en la búsqueda, ya que puede llegar un momento en que el niño se sienta perdido ante tanta información simultánea.

\subsubsection{Página de resultados}

\begin{tabular}{|l|l|}
\hline Formato de los registros & Sí \\
\hline Diferente etiquetado & Sí \\
\hline Formato de los ejemplares y situación de circulación & Sí \\
\hline
\end{tabular}

Tabla III. Prestaciones de búsqueda en página de resultados

En este apartado (tabla III) debe atenderse a la forma de presentación del registro, la estructuración de los elementos que se ofrecen y la pertinencia de la información bibliográfica.

De este modo, consideramos oportuno que se ofrezca, sobre todo, el registro en su formato abreviado. El formato expandido puede ser útil únicamente en determinados casos en los que se busque una edición concreta de un determinado ejemplar, lo que no suele ser muy frecuente entre este tipo de público. Abogamos por la utilización de las etiquetas siempre y cuando la terminología utilizada resulte explicativa, por lo que sería aconsejable abandonar la terminología ISBD en beneficio de términos comúnmente aceptados e identificativos. Por lo que respecta a su visualización, deben ajustarse a criterios admitidos por todos, como son que han de ir en mayúsculas, justificadas a la derecha y seguidas de dos puntos. Por supuesto, se han de evitar las abreviaturas. En definitiva, que resulten de fácil comprensión.

Por lo que se refiere al texto, sería aconsejable respetar los criterios de presentación establecidos para el común de los OPAC y que dicen que se debe justificar a la izquierda y evitar la separación de palabras entre líneas. La información del formato de los ejemplares y situación de circulación resulta muy útil.

\subsubsection{Gestión de contenidos}

En los experimentos más recientes sobre diseño de OPAC (tabla IV) se apuesta por la visualización de imágenes asociadas, lo que, en nuestro opinión, resulta importantísimo en el caso de catálogos en línea destinados al uso infantil. A los niños les resulta mucho más fácil el acceso a un documento si se permite su visualización total o parcial. 


\begin{tabular}{|l|c|}
\hline Consulta a diferentes catálogos & Sí \\
\hline Utilización de un catálogo concreto en diferentes momentos & No \\
\hline Gestión de distintos tipos de elementos informativos & Sí \\
\hline Visualización de imágenes asociadas & Sí \\
\hline Enlaces a documentos externos & No \\
\hline Soporte del protocolo Z 39.50 & No \\
\hline Enlaces hipertextuales entre elementos de distintas páginas & Sí \\
\hline
\end{tabular}

Tabla IV. Prestaciones de búsqueda en la gestión de contenidos

La consulta simultánea a los diferentes subcatálogos de la biblioteca y la gestión de distintos tipos de elementos informativos pueden serles útiles, al igual que la disposición de enlaces hipertextuales entre los elementos de las distintas páginas, porque permiten, en todo momento, la navegación por el catálogo. No obstante, no son necesarios los enlaces a documentos externos, la utilización de un catálogo concreto en diferentes momentos o la presencia del protocolo Z39.50, ya que suelen resultar muy complicados para este tipo de usuarios.

\subsubsection{Módulo de ayuda en línea}

Toda ayuda es poca cuando se trata de buscar la información, por lo que creemos necesaria su existencia. Su icono debe ser fácilmente reconocible, y ha de proporcionar diferentes niveles de ayuda, en los que deben primar la claridad y la organización de la información, evitando explicaciones demasiado largas que puedan inducir a confusión.

\subsection{La interfaz de usuario}

Como ya anunciamos al comienzo de nuestro trabajo, la interfaz constituye un elemento crucial en la comprensión del catálogo por parte del usuario, y mucho más si se trata de niños.

\subsubsection{Diseño}

Si es importante el papel que juega el diseño (tabla V) en los OPAC destinados a los adultos, mucho más en el caso de los dirigidos a los niños. Por esta razón, se han de tener en cuenta en primer lugar todos los aspectos que se refieren a la $\mathrm{vi}$ sualización, es decir, la utilización de colores vistosos, de una tipografía atractiva y legible, de iconos fácilmente reconocibles, etcétera. En segundo lugar, y también relacionado con el apartado anterior, hay que favorecer la facilidad de lectura del texto, utilizando para ello principalmente técnicas de contraste y realce y evitando en la medida de lo posible el uso de la barra de desplazamientos, que entrecorta la lectura.

Scire. $12: 1$ (en.-jun. 2006) 153-162. ISSN 1135-3761. 
Otros de los aspectos que hay que tener en cuenta dentro de este apartado es la agrupación en bloques según el contenido informativo del registro, así como la visualización constante de la estrategia de búsqueda, para evitar la desorientación del usuario y permitir la modificación en cualquier momento según las necesidades. Además, se valorará positivamente el resalte en los registros recuperados de los términos introducidos en la búsqueda y de aquellos elementos ya consultados.

Igualmente resulta aconsejable respetar la estructuración de la página en los tres niveles recomendados: superior para identificar la base de datos, el sistema de búsqueda utilizado y el título de la misma; intermedia para las opciones principales de búsqueda e inferior para el resto de opciones.

\begin{tabular}{|l|l|}
\hline Resalte de elementos & Sí \\
\hline Estructuración en tres niveles & Sí \\
\hline Agrupación en bloques de datos relacionados & Sí \\
\hline
\end{tabular}

Tabla V. Características de la interfaz: diseño

\subsubsection{Ergonomía}

Dentro de este punto destinado a la mejora del entorno laboral destacamos la posibilidad de seleccionar los registros en el caso de que el número de resultados sea múltiple (tabla VI).

En este apartado resultan altamente importantes la personalización del diseño y la adaptación del entorno a las preferencias del usuario. También es interesante que se ofrezca la posibilidad de establecer perfiles de usuario, por ejemplo atendiendo a los diferentes grupos de edades del público infantil que establezca la biblioteca.

Finalmente, sería muy positiva la integración con el módulo de circulación: facilidad para la gestión completa de préstamo, reservas, etcétera, para favorecer la autonomía del niño en la biblioteca.

No tendrían sentido en este caso otros aspectos muy valorados en la evaluación de catálogos para adultos, como son las posibilidades de descarga y el formato

\begin{tabular}{|l|c|}
\hline Posibilidad de seleccionar registros & No \\
\hline Formato de exportación de registros & No \\
\hline Posibilidad de descarga & No \\
\hline Personalización del diseño & Sí \\
\hline Integración con el módulo de circulación & Sí \\
\hline
\end{tabular}

Tabla VI. Características de la interfaz: ergonomía

Scire. $12: 1$ (en.-jun. 2006) 153-162. ISSN 1135-3761. 
de exportación de registros, opciones del sistemas que serían poco o nada utilizadas por el público infantil.

\subsubsection{Amigabilidad}

Los aspectos a evaluar dentro de esta característica (tabla VII), íntimamente ligada a la descripción de los catálogos en línea, son los siguientes: Primero, la sintaxis de los mensajes: estos han de ser simples y concisos. Se han de evitar, en la medida de lo posible, las abreviaturas, los tecnicismos, las oraciones negativas y la voz pasiva. Pueden existir varios tipos de mensajes: de error, informativos, explicativos... En segundo lugar, la terminología adecuada al usuario, valorando positivamente la no utilización de términos propios de la profesión. Otro aspecto sería la adecuación de iconos/botones, que cobra especial relevancia en los catálogos infantiles; en ellos debe primar la legibilidad y el carácter no redundante y autoexplicativo, así como su integración en el portal de la biblioteca. En último lugar estarían las sugerencias del sistema. Este ofrecerá al niño opciones automáticas que pondrán en evidencia el carácter interactivo del OPAC, es decir, ejemplos, indicaciones de modo automático de búsquedas fallidas, etcétera.

\begin{tabular}{|l|c|}
\hline Terminología adecuada al usuario & Sí \\
\hline Adecuación de iconos/botones & Sí \\
\hline Integración en acciones del sistema operativo & No \\
\hline Sugerencias del sistema & No \\
\hline
\end{tabular}

Tabla VII. Características de la interfaz: amigabilidad

\section{Conclusiones}

Partiendo de la base que el catálogo debe integrarse en el sistema de información global de la biblioteca y del centro en el que se circunscribe, nuestro trabajo tenía como único objetivo la realización de un patrón que permitiera posteriormente la evaluación de los catálogos en línea destinados a un público concreto: los usuarios infantiles.

Las necesidades de este tipo de usuario a la hora de realizar sus búsquedas en el OPAC varían sustancialmente respecto a las del público adulto, y esta salvedad ha sido tenida en cuenta en el diseño del modelo de evaluación de los catálogos infantiles propuesto. De ahí que en este caso no sean necesarios muchos de los parámetros útiles e imprescindibles cuando se evalúan los catálogos de adultos, como, por ejemplo, la posibilidad de exportar registros.

Centrándonos ya en el propio modelo de evaluación, diremos que este se divide en dos partes fundamentales: las variables que se estudian dentro de lo que se Scire. $12: 1$ (en.-jun. 2006) 153-162. ISSN 1135-3761. 
denominan prestaciones de búsqueda y las que se centran en las características de la interfaz.

En líneas generales, y resumiendo las conclusiones a las que hemos llegado después de la creación del modelo, podemos decir que el catálogo infantil "ideal" debería cumplir entre otras las siguientes premisas: es importante que incluya las características hipertextuales con las que el niño se encuentra más familiarizado; ha de contar con un lenguaje que sea entendible para los usuarios infantiles y, por tanto, que se ajuste a su "argot", así como con un formato de presentación atractivo para los niños.

\section{Referencias}

Borgman, Christine L. (1996). Why are online catalogs still hard to use? // Journal of the American Society for Information. 47:7 (1996) 493-503.

Dovey, Matthew (2000). So you want to build a Union Catalogue? // Ariadne. 23 (2000). URL: < http://www.ariadne.ac.uk/issue23/dovey/intro.html>. Consultado: 2005-06-15.

Lorenzo Ramírez, Nuria (1993). Una mirada al futuro: el acceso a la información en las bibliotecas y centros de recursos escolares, infantiles y juveniles. // Boletín de la Asociacion Andaluza de Bibliotecarios. (Septiembre 1993) 49-56.

Moscoso, P. (1998). Análisis y evaluación de catálogos automatizados de acceso público en entorno web. // Revista Española de Documentación Científica. 21:1 (1998) 409-416.

Rodríguez Bravo, B.; Alvite Díez, L. (2002). Evaluación de interfaces de OPAC universitarias implementadas con innOPAC millenium. // Jornadas Bibliotecarias de Andalucía (12 $2^{\text {as }}$, El Escorial, Madrid, 2002). 21-30.

Rodríguez Bravo, B.; Alvite Díez, L. (2004a). Evaluación de interfaces de OPAC universitarias implementadas con UNICORN en las universidades madrileñas. // Jornadas Españolas de Bibliotecas Digitales (Madrid, 2002). URL: <http://mariachi.dsi.upv.es/ jbidi/jbidi2002/Camera-ready/Sesion/S1-2.pdf>. Consultado: 2005-06-16.

Rodríguez Bravo, B.; Alvite Díez, L. (2004b). Propuesta metodológica de evaluación de interfaces de OPAC. INNOPAC versus MILLENIUM. // Revista Española de Documentación Científica. 27:1 (2004) 30-44.

Stevens, Norman D. (1998). The catalog of the future: a speculative essay. // Information, Technology and Libraries. 17:4 (1998) 183-187. 